\title{
Redefining the regularity effect
}

\author{
ALAN J. PARKIN \\ University of Sussex, Brighton, England
}

\begin{abstract}
This study reports two experiments that further explore the regularity effect in single-word pronunciation. Experiment 1 shows that regularity effects are found only with irregular words that are "true" exceptions (e.g., PINT, MONK, BROAD). Words that are irregular in terms of grapheme-to-phoneme correspondence rules, but either are regular in terms of a higher order correspondence rule (e.g., PALM, HEALTH) or possess a divergent, although reasonably common, correspondence (e.g., GLOVE, HEAD), produce response times similar to those produced by regular words. These results indicate that the regularity effect is restricted to a smaller set of words than previously has been thought. Experiment 2 examines the regularity effect when subjects are required to delay their responses by $1,500 \mathrm{msec}$; no difference between exception and regular words is found. This finding indicates that previous demonstrations of the regularity effect cannot be attributed to articulatory differences between exception and regular word samples. Theoretical accounts of the regularity effect are considered briefly.
\end{abstract}

There is now considerable evidence that the performance of simple laboratory-based reading tasks is influenced by spelling-to-sound regularity. In an early study, Baron and Strawson (1976) showed that word lists comprising "exception" words (i.e., those possessing an irregular spelling-to-sound correspondence-PINT, BROAD, BREAK, etc.) were read aloud more slowly than were equivalent lists of regular words. This essential finding has been replicated in more refined experimental designs in which pronunciation latencies to individual words have been measured (Glushko, 1979; Stanovich \& Bauer, 1978; Underwood \& Bargh, 1982).

The nature and implications of this "regularity effect" in pronunciation will be considered later. At this point, I am more concerned with the nature of spelling-to-sound regularity itself. Demonstrations of the regularity effect typically have employed Venezky's (1970) grapheme-tophoneme (GPC) rules as a basis for classifying words as regular or exceptional in their pronunciations. Venezky's survey described phonemic correspondence rules for each letter of the alphabet as well as for vowel digraphs and consonant digraphs. For each grapheme, Venezky's account provided a major correspondence and the various minor correspondences that also occur. It is important to note that Venezky did not describe correspondence rules for larger units of letters. The use of these rules to define exceptions and the consistency of the regularity effect suggests that GPC rules provide

I am very grateful to Phil Nixon and to Mark Souter for running Experiment 1. Thanks also go to Gordon Brown and Max Coltheart for useful comments. I am also indebted to the two anonymous reviewers for their comments and many important suggestions. Please address all correspondence to Alan J. Parkin, Laboratory of Experimental Psychology, University of Sussex, Brighton, BN1 9QG, England. reasonable basis for predicting the ease with which single words are pronounced.

The present paper examines whether the presence of a minor GPC within a word is a sufficient condition for the demonstration of regularity effects. However, a preliminary issue that must be considered is whether regularity effects arise because of visual differences between exception and regular words. In any sample of exception words, one will find a significant number that are also exceptional in their orthographic structure (e.g., SUEDE, EPOCH, YACHT). Since these "orthographically irregular" items were not excluded from the various studies demonstrating regularity effects, it is possible that this confounding influence may have partially or even wholly contributed to previously observed effects. This possibility was recently explored by Parkin and Graystone (1984). They distinguished between words that had exceptional pronunciations and unique orthographic structures (e.g., YACHT) and those that had exceptional pronunciations but regular orthographies (e.g., FEVER). Their results showed that pronunciation latencies for both types of word were longer than those for regular words. However, the regularity effect was much larger for exceptions with unique orthographies. This finding suggests that previous regularity effects may have been exaggerated by the confounding influence of uncontrolled orthographic factors but that a reliable regularity effect does occur when the sample of exception words used is restricted to those with regular orthographies.

If one examines words defined as having minor spellingto-sound correspondences in terms of the Venezky (1970) rules, it becomes apparent that some of the words could be considered regular in terms of some higher order rule, that is, a rule for pronouncing a unit of letters larger than a grapheme. This point is illustrated 
by the exception word sample used by Coltheart, Besner, Jonasson, and Davelaar (1979) in a lexical decision experiment. They included SIGN as an exception word because, according to the Venezky rules, this word contains a variety of minor correspondences when the word is considered grapheme by grapheme. However, if one assumes that a higher order spelling rule terminal IGN = [ain] exists (cf. ALIGN, BENIGN, etc.), then SIGN could be judged as regular. Another rule of this kind is terminal ALM $=[\mathrm{a}: \mathrm{m}]$, which enables us to conclude that BALM, CALM, and PALM are regular in their pronunciation. As a further example, consider the influence of " $W$ " on vowel correspondences: Venezky (1970, p. 112) noted that regular spelling-to-sound correspondences do not often apply to vowel sounds preceded by "W." Examination of these irregularities shows that some follow a consistent pattem if one considers larger letter units. Thus, WAR = [wo:] is a consistent correspondence (e.g., WARN, WART, WARP), as is WAN $=$ [won] (e.g., WANT, WAND, SWAN).

In addition, one can identify words that exhibit "mildly inconsistent" spelling patterns (see Wijk, 1966). These are words that contain patterns of letters for which there are two or more common correspondences. Examples of these are -USH as in PUSH and RUSH, -OVE as in MOVE, GLOVE, and TROVE, -EAD as in HEAD and BEAD, and $-O O D$ as in GOOD and FOOD. These mildly inconsistent words can be contrasted with "true" exceptions in which the spelling-to-sound correspondences are either unique or extremely unusual (e.g., PINT, VASE, DOSE, MONK).

The above discussion suggests that there are degrees of irregularity in English spelling-to-sound correspondence. Experiment 1 examined whether this distinction is relevant to demonstrations of the regularity effect. In Experiment 1, subjects were required to pronounce two types of irregular word, and the latencies produced to these items are compared with those for words whose pronunciation is entirely regular (e.g., PILL). The first category of irregular word will be referred to as "mildly inconsistent" (MI) and is composed of two types of word: (1) those that possess a minor correspondence at the GPC level but that can be considered regular if one considers a higher order rule (e.g., HEALTH) and (2) words that are based on a letter pattern that is commonly pronounced in more than one way, with the constraint that the pronunciation carried by the word is one that contains a miner correspondence according to Venezky (1970) (e.g., GLOVE). The procedure by which MI words were devised is summarized in the Appendix. The second category of irregular words is that of "exception" (E) words, which are defined as words with a unique or very unusual spelling-tosound correspondence (e.g., PINT, MONK). If the presence of a minor GPC is a sufficient condition for the emergence of spelling-to-sound regularity effects, then $E$ and MI words should both be pronounced more slowly than regular $(\mathbf{R})$ words.

\section{EXPERIMENT 1}

\section{Method}

Subjects. The subjects were 16 undergraduate volunteers from the University of Sussex.

Materials. Thirty exception (E) words were selected. Because of the known influences of orthographic regularity on pronunciation, exception words composed of unusual or unique letter sequences (e.g., TSAR, CHAOS) were excluded from the sample. For each exception word, a matched $R$ word was selected as a control. Each of these words was matched in terms of length, word frequency (Carroll, Davies, \& Richman, 1971-SFI), and initial phoneme. Thirty mildly inconsistent (MI) words were also selected, and for each of these, a matched regular word was selected according to the same criteria as those for $E$ words. To make the analysis clearer, $R$ words matched to $E$ words will be designated $R e$ and those for MI words R-mi. Concerning the frequency variable, the mean SFI was 49.7 (7.4) for E words, 48.7 (7.7) for R-e words, 51.1 (9.2) for MI words, and 49.3 (8.3) for R-mi words. This indicates a small frequency bias for both $\mathrm{E}$ and $\mathrm{MI}$ words, but analysis showed that this bias was not significant in either word set [E vs. Re, $t<1$; MI vs. R-mi, $t(29)=1.07]$. The resulting materials are shown in the Appendix along with an account of the criteria used to define MI words.

Procedure. The experiment was programmed on a PDP-11/40 computer interfaced with an M 6809 microprocessor. The subjects were seated in a soundproof cubicle such that they were facing a 9-in. video monitor. They were informed that they would be presented with a series of individual words and that they should pronounce each one as quickly as possible. All the words were presented in lowercase and subtended a visual angle of approximately $1 \mathrm{deg}$. Each trial began with the presentation of a word, which remained on the screen until the subject had pronounced it or the default value of $1,200 \mathrm{msec}$ had been reached. Pronunciation of the word activated a microphone connected to a voice key interfaced with the microprocessor, which recorded the pronunciation latency. The intertrial interval was $1,200 \mathrm{msec}$. The experiment consisted of 30 practice trials followed by two blocks (A and B) of 62 experimental trials (the first 2 trials were dummies). Block A consisted of half the $\mathrm{E}$ and MI words and their respective $\mathrm{R}$ words, whereas Block $B$ contained all the remaining items. Each block was arranged in a totally random sequence, except for the dummies. Half the subjects received Block $A$ followed by Block $B$, whereas the other half received the reverse. There was a $30-\mathrm{sec}$ interval between blocks.

\section{Results}

Mean pronunciation latencies for each of the four types of word were calculated; these data are shown in Table 1. Trials in which subjects made pronunciation

Table 1

Mean Pronunciation Times (in Milliseconds) for E, MI, and R Words in Experiment 1

\begin{tabular}{ccc}
\hline Type of Word & $\begin{array}{c}\text { Mean Pronun- } \\
\text { ciation Time }\end{array}$ & $\begin{array}{c}\text { Mean Error } \\
\text { Rate }\end{array}$ \\
\hline E & 654 & .94 \\
MI & 623 & .50 \\
R-e & 633 & .10 \\
R-mi & 630 & .10 \\
\hline
\end{tabular}

Note-E $=$ words with a unique or unusual spelling-to-sound correspondence; $M I=$ words that possess an irregular GPC but are regular in terms of a higher order rule or exhibit a divergent correspondence that is reasonably common; $R=$ words whose pronunciation is regular by any definition. 
errors, exceeded the response deadline (1,200 $\mathrm{msec})$, or accidentally activated the voice key were excluded. When a subject's response to a particular item was excluded, the response time produced to its counterpart was also excluded. Thus, if STEAK were mispronounced, the response to STING was also ignored, and vice versa. This procedure resulted in the exclusion of $3.6 \%$ of the data. The data were analyzed in a two-way analysis of variance with word type $(E+R-e$ vs. $M I+R-m i)$ and trial type (E + MI vs. R-e + R-mi) as fixed factors. Two sets of scores were computed for each condition, one by collapsing across subjects and the other by collapsing across words. This enabled the calculation of min $F^{\prime}$ for each of the observed effects (Clark, 1973). The individual word means derived for this analysis are shown in the Appendix.

The analysis showed a main effect of word type in the by-subjects analysis $[F(1,15)=29.1, \mathrm{p}<.0001]$, but this just failed to reach significance in the by-words analysis $[F(1,58)=3.97, .05<p<.1]$. More importantly, the interaction was significant $\left[\min \mathrm{F}^{\prime}(1,66)=\right.$ $6.21, \mathrm{p}<.025]$. This interaction was analyzed by making separate comparisions of the difference between $\mathrm{E}$ words with their matched $\mathrm{R}$ words and $\mathrm{MI}$ words with their $\mathrm{R}$ words. Pronunciation latencies to $\mathrm{E}$ words were significantly longer than their matched $\mathrm{R}$ words $\left[\min F^{\prime}(1,68)=6.25, p<.025\right]$, whereas the equivalent comparison for MI words was nonsignificant. ${ }^{1}$

Errors were too few for any analysis, but a summary of the error pattern is shown in Table 1.

\section{EXPERIMENT 2}

Experiment 2 examined a methodological point concerning the delay in pronouncing exception words relative to regular words in Experiment 1. Although care was taken to equate these two groups of items on a variety of pertinent matching criteria, it was possible that the effects observed could have stemmed from differences in the ease with which exception and regular words can be articulated that are independent of factors that control initial access to correct pronunciation. Accordingly, it was decided to run an experiment in which subjects were given sufficient time to access the pronunciation prior to their being asked to overtly produce it. In this way, it was hoped that any difference in articulatory difficulty per se between exception and regular words would be revealed. Alternatively, if no regularity effect were obtained under these conditions, this would rule out the suggestion that the regularity effect obtained in Experiment 1 arose from uncontrolled differences in the articulatory properties of the two classes of words.

\section{Method}

Subjects. Ten subjects drawn from the technical staff of Sussex University and a visiting Open University summer school served as the subjects.
Materials. These were the same as the E and R-e items used in Experiment 1, except that one instance of "HITCH" was replaced by "HEDGE."

Procedure. The experiment was run using the same apparatus as that used in Experiment 1. On each trial, the subjects were first presented with a single word, which remained in view for $1,500 \mathrm{msec}$. Offset of the word was followed by a delay of $500 \mathrm{msec}$, after which "XX" appeared. The subjects were instructed to identify the word presented on the first part of the trial and then to pronounce it as quickly as possible when the "XX" pattern appeared. Pronunciation latency was measured from the onset of the "XX" pattern. The experiment was run in two blocks of 30 trials preceded by a practice block. Block 1 contained half of the $\mathrm{E}$ and their matched R-e words, and Block 2 contained the remainder. A "dummy" trial was placed at the head of each block.

\section{Results}

Mean correct pronunciation latencies were calculated in the same manner as those in Experiment 1. E words produced a mean latency of $443 \mathrm{msec}(\mathrm{SD}=84.1)$ and R-e words a mean of $444 \mathrm{msec}$ (75.4), a difference that was totally nonsignificant. In total only five errors were recorded.

\section{Discussion}

The results of Experiment 2 show that there is no regularity effect in pronunciation when subjects are allowed a delay between item presentation and pronunciation response. This result argues against an explanation of the regularity effect in terms of articulatory differences between $E$ and R-e words and suggests instead that the locus of the effect lies in processes that occur prior to the production of articulation. Explanations of this effect will now be considered.

Explanations of the redefined regularity effect. The first explanation of the regularity effect is based on the "two-route" model (e.g., Coltheart, 1978), which assumes that the pronunciation of a word can be derived in two different ways. The first of these can be termed the "lexical lookup" route, whereby the visual characteristics of the letter string are mapped onto the appropriate lexical entry from which the pronunciation response is then derived. The second can be termed the "phonological recoding route" and involves the translation of the visual code into a phonemic code by means of phonemic transcription rules. This latter route could be used in the pronunciation of nonwords, since these have no lexical entry. Regularity effects in pronunciation can be explained by assuming that these two routes operate in parallel to produce an overt response. Regular words present no problem, since both the lexical and phonological recoding routes produce the same response-the assumption being that the phonemic correspondence rules operate by applying the most common correspondence. Exception words, however, produce the correct response via the lexical route, but if the recoding route is able to produce a response, it will be incorrect. As a result, there is an incompatibility between the two response sources that must be resolved. This resolution, which might involve some recheck of the activated 
lexical representation, takes time, thus producing a delay in the pronunciation of exception words or, if the recoding route output is dominant, a pronunciation error in the form of a "regularization." Since pronunciation errors are relatively infrequent, it would seem that the recoding route is rarely dominant, and examination of those items producing these errors shows that they are mainly low frequency. This suggests that pronunciation errors originate when there is an above-average delay in producing a response via the lexical route, a delay that one might expect with low-frequency words.

Experiment 2 also provided support for the view that the regularity effect arises due to some form of conflict prior to articulation. Here, the regularity effect was not found when subjects were given a delay between identification and response. One can suggest that the delay provided sufficient time for any conflict about pronunciation to be resolved, hence removing the basis of the regularity effect.

The alternative view of pronunciation is that forwarded by Glushko (1979) and subsequently advocated by Kay and Marcel (1981). The basic idea of Glushko's "activation and synthesis" model is best explained in his own words:

As letter strings are identified, there is parallel activation of orthographic and phonological knowledge from a number of sources in memory. This knowledge may include the stored pronunciation of the letter string, pronunciation of words that share features with the letter string, and information about the spelling-to-sound correspondence of various subparts of the letter strings. A pronunciation is generated using a procedure for determining how to modify the activated information in order to synthesize the desired articulatory program. (p. 678)

Within Glushko's model the regularity effect is assumed to arise because exception words produce conflicting sources of information about phonemic correspondence. However, to explain how this conflict is resolved, one must be more precise about the "procedure" that determines the "desired" pronunciation. By any standard, the word PINT is exceptional in its pronunciation. The correspondence INT = [aint] is unique, and there are nine words exhibiting the regular correspondence (e.g., TINT, HINT). Given this imbalance of information, one would expect PINT to be mispronounced quite often when 'speeded responding is required. Yet PINT, like the majority of exception words, is rarely mispronounced (not at all in Experiment 1). To account for this, it is necessary to suggest that phonemic information provided by an activated lexical entry has greater salience than that provided by other informational sources. With this modification, Glushko's activationsynthesis model becomes an implicit two-route model in which two potential sources of phonemic information are distinguished. Admittedly, this distinction is in terms of salience, as opposed to processing structures, but it nonetheless ensures that experiments with the pronunciation of exception words will fail to provide an unequivocal test of two-route versus activation-synthesis accounts of pronunciation.

The absence of any difference in pronunciation between $\mathrm{MI}$ and $\mathrm{R}$ words is therefore of considerable importance in evaluating the merits of these two contrasting approaches to pronunciation. Concerning a two-route view of pronunciation, it is important to note that the failure to find any difference between MI and R-mi words argues against a phonological recoding route operating solely on the basis of GPC's applying the major correspondence. If this were so, then all the MI words should have been wrongly encoded (e.g., HEALTH $\rightarrow[$ hi:l $\theta]$, HOOK $\rightarrow[h u: k])$, resulting in pronunciation delay. Since this did not occur, a modified version of the two-route idea must be offered.

As a starting point, one could suggest that the phonological recoding route possesses information about higher order letter-to-sound correspondences. This assumption would explain why words such as HEALTH, SIGN, and PALM cause no problem, since correct pronunciation could be achieved by assigning greater salience to the correspondence derived from the higher order rule (e.g., H + EALTH would be preferred to $\mathrm{H}+\mathrm{EA}+\mathrm{L}+\mathrm{TH})$. Although this "weighted rule" theory could explain the pronunciation of some MI words, how it could be modified to explain the general ease with which MI words are pronounced is not clear. How, for example, could a rule-based procedure choose between the equipotential pronunciations of USH or OVE?

Adding higher order correspondence rules to the phonological route is therefore not sufficient to account for lack of an MI-R-mi difference. In addition, it must be argued that the rules embody information about letter patterns in English that have more than one common correspondence; thus, the rules might register the fact that -OVE has three common correspondences. Rules of this type, when presented with D + OVE, would indicate three possible correspondences for -OVE but, in the absence of lexical information, have no basis for selecting the appropriate one. Furthermore, assume that presentation of a letter string results in an attempt to derive its pronunciation by both the lexical and the phonological recoding routes. Providing that factors such as orthographic regularity and word frequency are held constant, there is no reason to suppose that $\mathrm{E}, \mathrm{MI}$, and $\mathrm{R}$ words would differ in the speed with which they accessed their lexical representations and pronunciations. Similarly, E, MI, and R words should not differ in the speed at which they are engaged by the phonological route. In the case of $\mathrm{R}$ words, the application of spelling-to-sound rules would be sufficient to produce the correct pronunciation. For MI words, however, these rules would indicate multiple pronunciations, which would delay or prevent the production of a pronunciation. Thus, it is unlikely that the encoding route could produce a phonemic translation in advance of that offered by the lexical route; thus, the pronuncia. 
tion of MI words proceeds on the basis of lexical information alone. Slower pronunciation latencies for $\mathrm{E}$ words can be explained by supposing that the phonological recoding rules do not extend to the more unusual correspondences displayed by these words. As a result, these words would be processed as if they were regular (i.e., PINT would be assigned [pint]). On a significant number of occasions, production of this aberrant response would coincide with the output of the lexical route. This would result in a discrepancy that would have to be resolved and would thus account for the significant delay in the pronunciation of $E$ words.

Glushko's (1979) "activation-synthesis" model has some difficulty in accounting for the similar pronunciation times for MI and R words. By Glushko's definition, $84 \%$ of the words in the MI sample are inconsistent (i.e., they have visual "neighbors" that exhibit alternative pronunciations), yet these words show no evidence of delayed pronunciation when compared with the $R$ words, which are all consistent. According to this model, all words that activate conflicting information about phonemic correspondence should show some delay in pronunciation (the so-called "consistency effect"), but no such effect is apparent.

The argument developed above suggests that Glushko's (1979) model has difficulty in accounting for the absence of any difference in pronunciation latency between $\mathrm{MI}$ and $\mathrm{E}$ words. However, there is a potential modification of the model that could account for this finding. The activation-synthesis model predicts that both E words (PINT) and MI words (GLOVE) should produce longer pronunciation latencies than regular words because both types have inconsistent neighbors that produce conflicting information about pronunciation. However, since only exception words slow pronunciation, one might question whether the degree of conflict is comparable in $\mathrm{E}$ and MI words. If one considers the issue in terms of the proportion of types ${ }^{2}$ representing the conflicting pronunciation, it is clear that $\mathrm{E}$ and $\mathrm{MI}$ words differ; for $\mathrm{E}$ words, the proportion is very small (e.g., PINT, 1/10), whereas for MI words the proportion is, by definition, higher (e.g., GLOVE, $5 / 13$ ). If the degree of conflict is determined by the relative strengths of the competing pronunciations compared with the activated lexical entry and its con sistent neighbors, then $\mathrm{E}$ words, when the competition is highest, will engender the most conflict. Even so, the fact that mispronunciations of $\mathrm{E}$ words are relatively uncommon indicates that lexical activation has particular salience in determining the eventual response. For MI words, the relative strengths of the competing pronunications is less; this, in conjunction with the greater salience of the activated lexical entry, allows the competing information to be overcome more easily and results in less pronunciation delay. However, a weakness of this explanation is that it fails to account for the lack of any difference between MI and R-mi words; MI words should still activate conflicting sources of infornation about pronunciation, which, in turn, ought to cause a delay. At present, this could be resolved only by arguing that the pronunciation latency is insensitive to the smaller delays caused by MI words.

\section{CONCLUSION}

This study has demonstrated that the regularity effect in pronunciation is restricted to words that have exceptional pronunciations. Words that have pronunciations consistent with some higher order rule or divergent pronunciations of letter patterns commonly pronounced in more than one way produce pronunciation latencies similar to those produced by regular words. At present, both existing models of pronunciation can be modified to account for this redefined regularity effect, so we must await future experiments to determine which model offers the most satisfactory account.

\section{REFERENCES}

Baron, J., \& Strawson, C. (1976). Use of orthographic and word-specific knowledge in reading words aloud. Journal of Experimental Psychology: Human Perception and Performance, 2, 386-393.

Carroll, J. B., Davies, P., \& Richman, B. (1971). The American Heritage word frequency book. Boston: Houghton-Mifflin.

Clark, H. H. (1973). The language-as-a-fixed-effect fallacy: A critique of language statistics in psychological research. Journal of Verbal Learning and Verbal Behavior, 12, 335-359.

Coltheart, M. (1978). Lexical access in simple reading tasks. In G. Underwood (Ed.), Strategies of human information processing. London: Academic Press.

Coltheart, M., Besner, D., Jonagson, J. T., \& Davelaar, E. (1979). Phonological recoding in the lexical decision task. Quarterly Journal of Experimental Psychology, 31, 489-508.

GuUshкo, R. (1979). The organization and activation of orthographic knowledge in reading aloud. Journal of Experimental Psychology: Human Perception and Performance, 5, 674-691.

KAY, J., \& MARCEL, A. (1981). One process, not two, in reading words aloud: Lexical analogies do the work of non-lexical rules. Quarterly Journal of Experimental Psychology, 33A, 397-413.

PARKIN, A. J. (1984). Experiments on the nature of the consistency effect in single word pronunciation. Manuscript submitted for publication.

Parkin, A. J., \& Graystone, D. (1984). Orthographic versus phonological irregularity in the pronunciation of single words. Manuscript submitted for publication.

Stanovich, K. E., \& Bauer, D. W. (1978). Experiments on the spelling-to-sound irregularity effect in word recognition. Memory \& Cognition, 6, 410-415.

UNDERWOOD, G., \& BARGH, K. (1982). Word shape, orthographic regularity and contextual interactions in a reading task. Cognition, 12, 197-209.

VENEZKY, R. L. (1970). The structure of English orthography. The Hague: Mouton.

WiJk, A. (1966). Rules of pronunciation for the English language. London: Oxford University Press.

\section{NOTES}

1. Although the MI and $\cdot R-m i$ words did not show a significant difference in frequency, it was noted that the difference was larger than that for the E-Re comparison (1.8 vs. $1.0 \mathrm{SFI}$ units). It was therefore decided to perform an additional check that the lack of an MI-R-mi difference was not attributable to MI words' being of higher frequency, which, in turn, might compensate for any delay in pronunciation latency. Accordingly, the data were reanalyzed minus any pair of items in which the 
MI word was more than 6 SFI units more frequent than the $\mathrm{R}$-mi word. When the reverse was found, that is, $\mathrm{R}$-mi was more frequent than MI by more than 6 units, the pair was left in. This procedure reduced the word set to 25 pairs, with a mean difference (MI-R-mi) of -.99 SFI units; thus, in this revised sample, the R-mi words tended to be the more frequent. This reanalysis yielded mean pronunication latencies of $630 \mathrm{msec}$ for MI words and $631 \mathrm{msec}$ for R-mi words.

2. The decision to discuss conclict in terms of types rather than in terms of tokens is based on Parkin's (1984) finding that nonword pronunciation is influenced by types rather than by tokens. He found that nonwords based on a letter pattern whose mispronounced form accounts for a large number of the tokens in the language (e.g., BREAT, -EAT in GREAT accounts for $64 \%$ of the -EAT tokens) do not produce delays in pronunciation. However, nonwords based on letter patterns with at least two families of differently pronounced types (e.g., BOVE) did produce delays.

\section{APPENDIX}

Table 2

Materials and Individual Pronunciation Latencies (in Milliseconds) in Experiment 1

\begin{tabular}{|c|c|c|c|c|c|c|c|}
\hline \multicolumn{8}{|c|}{ Word Type } \\
\hline $\mathrm{E}$ & Latency & Re & Latency & MI & Latency & R-mi & Latency \\
\hline vase* & 655 & vent & 619 & hook & 620 & hike & 622 \\
\hline threat & 689 & throat & 632 & wasp & 608 & wipe & 596 \\
\hline dose & 632 & daze & 599 & wealth & 610 & wither & 638 \\
\hline lever & 629 & level & 621 & hood & 620 & heal & 664 \\
\hline monk & 676 & mist & 640 & worm & 670 & wink & 646 \\
\hline pint & 678 & pill & $\begin{array}{l}070 \\
578\end{array}$ & palm & 679 & pain & $\begin{array}{l}040 \\
621\end{array}$ \\
\hline clerk & 637 & click & 607 & swap & 635 & swig & 703 \\
\hline bear & 585 & boar & 665 & squat & 695 & squid & 742 \\
\hline sieve & 717 & surge & 694 & blood & 611 & block & 597 \\
\hline bowl & 656 & belt & 594 & flood & 692 & float & 669 \\
\hline sweat & 676 & swing & 694 & watch & 550 & wheel & 609 \\
\hline broad & 636 & brief & 611 & warp & 696 & weed & 638 \\
\hline route & 628 & rinse & 613 & shoe & 584 & shed & $\begin{array}{l}020 \\
577\end{array}$ \\
\hline toll & 648 & tank & 624 & earn & 615 & ease & 588 \\
\hline marine & 691 & marble & 638 & head & 602 & help & 577 \\
\hline hover & 674 & hitch** & 626 & wart & 592 & wail & 592 \\
\hline demon & 701 & delta & 688 & love & 566 & link & 622 \\
\hline fever & 652 & foggy & 673 & swallow & 629 & swindle & 670 \\
\hline lager & 677 & latin & 579 & rook & 601 & roll** & 587 \\
\hline naked & 594 & nutty & 625 & wand & 632 & weld & 659 \\
\hline halve & 617 & hitch & 617 & bull & 599 & boil & 601 \\
\hline breast & 654 & breeze & 616 & bush & 605 & bulb & 605 \\
\hline steak & 696 & sting & 718 & dread & 650 & dream & 629 \\
\hline pour & 677 & pipe & 605 & sign & 631 & side & 647 \\
\hline soul & 689 & seed & 671 & swarm & 734 & swish & 672 \\
\hline break & 598 & brain & 596 & word & 573 & wilt & 617 \\
\hline gross & 648 & grace & 630 & money & 595 & minor & 625 \\
\hline gruss & 619 & green & 592 & glove & 605 & glide & 637 \\
\hline soot & 658 & seep & 686 & dove & 633 & dope & 614 \\
\hline touch & 605 & truck & 625 & health & 585 & hammer & 643 \\
\hline
\end{tabular}

Note $-E=$ words with unique or unusual pronunciations; $R \cdot e=$ words with regular pronunciations matched to $E$ words; $M I=$ words with regular pronunciations according to a higher order rule or exhibiting a divergent correspondence that is reasonably common; $R-m i=$ words with regular pronunciations matched to MI words. "American readers should note: In England, "vase" rhymes with "cars," "lever" is pronounced as in "Lever Brothers," and "hover" is pronounced [hova] . **Due to an experimental error, "hitch" was repeated and "roll" was included as an $R$ word. Given the substantial nature of the findings, these items were not discounted from the analysis.

\section{Rules for Defining MI Words}

HOOK, ROOK: Unit OOK most frequently corresponds to [uk]. WASP, WATCH, SWAP, SWALLOW, WAND: WA most frequently corresponds to [wo].

WEALTH, HEALTH: These are consistent with the rule EALTH $=[\mathrm{el} \theta]$.

HOOD: $O O D=[\mathrm{ud}]$ is one of three common correspondences. WORM, WORD: WOR is most frequently pronounced [wa:] PALM: This is consistent with the rule terminal ALM $=[\mathrm{a}: \mathrm{m}]$. SQUAT: This is consistent with the rule SQUA $=$ [skwo]

BLOOD, FLOOD: OOD $=[\wedge d]$ is one of three common correspondences.

WART, WARM, SWARM, WARD: These are consistent with the rule WAR $=[$ wo: $]$.
SHOE: Terminal OE $=[\mathrm{u}]$ is reasonably frequent. uent. EARN: EAR $=[\theta:]$ is as common as EAR $=[i \theta]$. HEAD, DREAD: EAD most commonly corresponds to [ed]. LOVE, GLOVE, DOVE: OVE $=[\wedge \mathrm{A}]$ is one of three common correspondences.

BULL: [ul] is one of two common correspondences for ULL. BUSH: [uf] is one of two common correspondences for USH. SIGN: This is consistent with the rule terminal IGN = [ain]. MONEY: Terminal ONEY $=[\wedge n i]$ is the most common correspondence.

(Manuscript received April 6, 1983; revision accepted for publication January 18,1984 .) 